\title{
Mortality following acute medical hospitalization in Denmark - a population-based cohort study
}

\author{
Betina Hansen ${ }^{1,3^{*}}$, Morten Schmidt ${ }^{1}$, Sussie Antonsen ${ }^{1}$, Jens Møller ${ }^{2}$, Carsten Thordal ${ }^{2}$, Henrik Toft Sørensen ${ }^{1}$ \\ From Danish Society for Emergency Medicine: Research Symposium 2010 \\ Roskilde, Denmark. 20-21 May 2010
}

\begin{abstract}
Background
The age of the general population is increasing: It is estimated that the population aged above 65 years will increase by 400,000 over the next 30 years. A third of this increment is comprised by persons older than 80 years. $45 \%$ of the general population and $88 \%$ of the population aged above 65 years have at least one chronic disease. Thus, aging of the population is expected to cause an increase in morbidity and an increase in number of hospitalizations. The increasing number of hospitalizations will therefore involve an increasing number of patients with more than one chronic disease. The aim was to examine 1-, 3- and 6month mortality after acute medical hospitalization according to sex, age, and comorbidity.
\end{abstract}

\section{Methods}

Using data from the Danish National Registry of Patients, we conducted a population-based cohort study including all patients with an admission in 2008 to the Medical Admission Unit (MAU) at Aarhus University Hospital-Nørrebrogade. Only first-time admissions were included. The patients were followed until death, emigration, or 6 months after date of admission using data from the Civil Registration System. Using discharge diagnoses registered before 2008, we ascertained comorbidity and defined three levels of comorbidity indexscore (low, medium, high). We examined mortality according to sex, age and comorbidity.

\section{Results}

We identified 4,494 patients with a first-time admission in 2008 to MAU. 3,768 (84\%) were classified as acute, $252(6 \%)$ as non-acute, and $474(11 \%)$ were unclassified.
Among the patients with acute admissions, 54\% were women and $45 \%$ men. The median age was 63 years for women and 60 for men. $45 \%$ of the patients had a low comorbidity index-score, $33 \%$ had a medium indexscore and $22 \%$ had a high index-score. Overall mortality was $5.5 \%$ after 30 days, $9.3 \%$ after 90 days, and $12.6 \%$ after 6 months. Mortality of patients older than 80 years was $13.9 \%, 23.4 \%$, and $29.5 \%$, respectively. Patients with the high comorbidity index-score had a mortality of $11.9 \%, 20.4 \%$, and $26.9 \%$, respectively.

\section{Conclusion}

Mortality after admission with an acute medical disease was strongly associated with age and comorbidity, but did not vary by sex.

\section{Author details}

'Department of Clinical Epidemiology, Aarhus University Hospital, Denmark. ${ }^{2}$ Department of Endocrinology and Internal Medicine, Aarhus University Hospital-Nørrebrogade, Denmark. ${ }^{3}$ Center of Emergency Medicine Research, Aarhus University Hospital, Denmark.

Published: 17 September 2010

doi:10.1186/1757-7241-18-S1-P21

Cite this article as: Hansen et al:: Mortality following acute medical hospitalization in Denmark - a population-based cohort study. Scandinavian Journal of Trauma, Resuscitation and Emergency Medicine 2010 18(Suppl 1):P21.

* Correspondence: bh@dce.au.dk

${ }^{1}$ Department of Clinical Epidemiology, Aarhus University Hospital, Denmark

Full list of author information is available at the end of the article 